\title{
Cloning of a new glutathione peroxidase gene from tea plant (Camellia sinensis) and expression analysis under biotic and abiotic stresses
}

\author{
Jian-Yu Fu
}

\begin{abstract}
Background: Tea plant, Camellia sinensis (L.) O. Kuntze, a well-known heavy metal hyperaccumulator, possesses a powerful tolerance to heavy metals. The heavy metal stresses lead to reactive oxygen species (ROS) production, and high concentration of ROS is harmful to plants. The glutathione peroxidase gene has positive function to damage induced by ROS. To understand the mechanism of tolerance to deferent stresses in tea plant, a new glutathione peroxidase gene of tea plant was cloned and its expression pattern was analyzed under abiotic and biotic stresses.

Results: A novel CDNA encoding glutathione peroxidase of tea plant (Camellia sinensis) was isolated by rapid amplification of cDNA ends (RACE) method and designated as CsGPX2 (GenBank Accession No. JQ247186). This full-length sequence was 917 nucleotides including a 510 bp open reading frame (ORF), which encoded a polypeptide of 169 amino acids. The deduced amino acid sequence showed high homology with glutathione peroxidases of angiosperms and contained the characteristic conserved motifs of ILAFPCNQF and FTVKD, the highest level of similarity was $85 \%$ to a glutathione peroxidase from Ricinus communis (Accession NO. XP_002509790.1). Tissue expression pattern analysis indicated that CsGPX2 expressed similarly in root, stem, leaf and flower of tea plant. The CsGPX2 gene showed strong responses to most abiotic stresses including salinity, heavy metal toxicity, drought, heat, plant hormones, but could not be induced by biotic treatment.
\end{abstract}

Conclusions: The result suggested that CsGPX2 had potential function in protecting tea plant from peroxidative damage induced by some abiotic stresses.

Keywords: Abiotic stresses; Biotic treatment; Glutathione peroxidase; Induced; Tea plant

\section{Background}

In plants, aerobic reactions lead to reactive oxygen species (ROS) production such as superoxide radical $\left(\cdot \mathrm{O}_{2}^{-}\right)$, hydroxyl radical $(\cdot \mathrm{OH})$, and hydrogen peroxide $\left(\mathrm{H}_{2} \mathrm{O}_{2}\right)$. It has been proved that ROS has two entirely different roles in plants, signal function and oxidative damage. When the ROS concentration is at an acceptable low level, they play an important signaling function in plants controlling processes such as growth, development, response to biotic and abiotic environmental stimuli, and programmed cell death (Bailey-Serres and Mittler 2006. While high concentration of ROS are harmful to cells (Rodríguez Milla et al. 2003; Navrot et al. 2006; Ramos

Correspondence: mybatigoal@mail.tricaas.com

Tea Research Institute, Chinese Academy of Agricultural Sciences, 9 South Meiling Road, Hangzhou 310008, China et al. 2009), and consistent accumulation of ROS imposes ultimately oxidative stress, exacerbating cellular damages (Bhattacharjee 2012).

Under biotic or abiotic stress, ROS may dramatically accumulate in plants and generate oxidative damage (Faltin et al. 2010; Gill and Tuteja 2010; Suzuki et al. 2011). On the other side, plants have developed some enzymatic systems including superoxide dismutases (SOD), catalases (CAT), ascorbate peroxidases (APX), peroxiredoxins and non-enzymatic mechanisms to protect against oxidative damage caused by these ROS (Agrawal et al. 2002; Navrot et al. 2006; Anjum et al. 2012). Glutathione peroxidases (GPXs) catalyze the reduction of $\mathrm{H}_{2} \mathrm{O}_{2}$, organic hydroperoxides, and lipid peroxides using GSH and/or other reducing equivalents (Ursini et al. 1995).

The study of GPXs in plants followed the previous research in mammals (Criqui et al. 1992; Bae et al. 2009). 
Since the first plant GPX cDNA was isolated from a wild tobacco (Nicotiana sylvestris) (Criqui et al. 1992), a significant part of novel genes for GPXs were reported in succession. To date, more than 100 GPXs were isolated from diverse plants and even 8 GPXs in Arabidopsis were cloned (Gaber et al. 2012). These GPX genes from plants were clustered in five main groups (Holland et al. 1993; Jung et al. 2002). Clades I and II are hypothesized to contain, respectively, chloroplastic and cytosolic isoforms; clades III and IV, both cytosolic and secreted proteins; and clade V, cytosolic proteins and proteins with $\mathrm{N}$ terminal transit peptides for targeting either to the mitochondria or to both the mitochondria and chloroplasts (Margis et al. 2008; Ramos et al. 2009). Most of the plant GPXs show high similarity to animal phospholipid hydroperoxide glutathione peroxidases (PHGPXs) (Rodríguez Milla et al. 2003), but their structure, substrate specificities, and subcellular localization were large different with mammal GPXs (Miao et al. 2005; Miao et al. 2006; Yang et al. 2006).

Tea, Camellia sinensis (L.) O. Kuntze, originated in China, is one perennial woody evergreen plant. Tea plant, a heavy metal hyperaccumulator, cumulates heavy metals by uptake of them from soil and air consistently. Meanwhile, it possesses a corresponding tolerance to heavy metals (Feng et al. 2009; Anjum et al. 2012; Hossain et al. 2012). To date there is only one glutathione peroxidase gene (GenBank Accession No. AEC10977) was isolated from tea plant but no function research was involved. In the present study, another new glutathione peroxidase gene of tea plant was cloned and expression pattern was analyzed under simulated environmental conditions, plant hormones, herbivore damage. The result showed that the gene was induced by all abiotic stresses dramatically, but the gene was not sensitive to biotic treatment, and these findings may help us to understand the mechanism of tolerance to deferent stresses in tea plant.

\section{Methods}

\section{Plants materials}

Tea plants, Camellia sinensis. cv. Longjing 43 were cultured in vermiculite and kept in light incubator under controlled conditions $\left(25^{\circ} \mathrm{C}\right.$ and $10 / 14 \mathrm{~h}$ light/dark photoperiods) with $85 \%$ relative air humidity. Three-week-old seedlings were treated with all biotic and abiotic stresses, the harvested organs (roots, stems and leaves were collected from seedlings, flowers were picked from flowering field plants) were immediately frozen in liquid nitrogen and stored at $-80^{\circ} \mathrm{C}$ until nucleic acid were extracted. For heavy metals, salinity and drought stresses, roots of 10 intact plants were partly soaked in $200 \mu \mathrm{M} \mathrm{FeSO}, 200 \mu \mathrm{M}$ $\mathrm{CuSO}_{4}, 500 \mathrm{mM} \mathrm{NaCl}$ and $500 \mathrm{mM}$ mannitol for 6 hours (Rodríguez Milla et al. 2003; Miao et al. 2006). For heat treatment, seedlings were kept under $40^{\circ} \mathrm{C}$ in chambers for 3 hours. For plant hormones treatments, seedlings were sprayed 2-3 times in 12 hours with $1 \mathrm{mM}$ SA (salicylic acid), ABA (abscissic acid), GA (gibberellin), NAA (naphthaleneacetic acid) and MeJA (Methyl jasmonate) (Sigma-Aldrich, St Louis, MO, USA) solutions under continuous light (Navrot et al. 2006; Rodríguez Milla et al. 2003; Miao et al. 2006). Control seedlings were treated with deionized water. MeJA, GA and NAA was dissolved in sterilized water with $2 \%$ ethanol, SA and ABA were dissolved in sterilized water at an ultimate concentration of $1 \mathrm{mM}$. For biotic treatments, two larvae of tea geometrids (Ectropis obliqua Prout) starved for $24 \mathrm{~h}$ were placed on the foliage for feeding damage, and the damaged leaves were harvested after 6, 12, 24, 48, 72 and 96 hours. Leaves of intact plants were collected as control at the time the treatments started.

\section{The cDNA cloning and sequence analysis}

Total RNA were isolated with a polysaccharide and polyphenol total RNA isolation kit (BioTeke, Beijing, China). The quality and concentration of the RNA were checked by NanoDrop 1000 spectrophotometer (Thermo Fisher Scientific, Waltham, MA, USA) and formaldehyde agarose gel electrophoresis. Total RNA was reverse transcribed to the first-strand cDNA with an oligo $(\mathrm{dT})$ primer designed with an adaptor sequence according to the protocol of the SMART RACE cDNA Amplification Kit (Clontech, Mountain View, CA, USA.). The RACE PCR primers was designed and synthesized based on the sequence obtained from a cDNA library (a lab internal source) of tea plants. Primers of CsGPX2-5'RACE (5'-ATCTGTTCACGAGTT CACCGTCAAGG-3') and universal primer A mix (UPM, long: 5' -CTAATACGACTCACTATAGGGCAAGCAGTG GTATCAACGCAGAGT-3'; short: 5'-CTAATACGACTC ACTATAGGGC-3') were used to carry out the $5^{\prime}$ RACEPCR under the recommended condition by the kit. The 3' RACE-PCR were performed with the 3'-RACE CDS Primer A (5' -AAGCAGTGGTATCAACGCAGAGTAC (T)30 N ${ }_{-1}$ N-3' and specific primer CsGPX2-3'RACE:5' GGTGGATTTTTTGGTGATGGAA-3') under the same condition as above-mentioned. The PCR products was purified by kit and subcloned into the pGEM-T Easy vector (Promega Corporation, Madison, WI, USA), transferred into $E$. coli $\mathrm{DH} 5 \alpha$ and sequenced bidirectionally by ABI 3730 automated sequencer (Applied Biosystems, Foster City, CA, USA) with the universal primers of M13.

The two RACE products and original fragment were automatically aligned and assembled by DNAMAN program, and the full-length cDNA sequence of CsGPX2 gene was obtained by splicing. The deduced amino acid sequence comparison was performed via BLAST program (NCBI, National Center for Biotechnology Services, http:// www.ncbi.nlm.nih.gov). The CsGPX2 and other plants glutathione peroxidase genes retrieved from GenBank were 
aligned with online CLUSTAL W (http://www.ebi.ac.uk/ Tools/msa/clustalw2). The phylogenetic tree was constructed by MEGA 4 program based on the converted result from CLUSTAL W alignments.

Expression analysis in organs and under different stresses The $C s \mathrm{GPX}_{2}$ expression profiles in different organs (roots, stems, leaves and flowers) of tea plant and under biotic and abiotic treatments were investigated by real time qRT-PCR. Total RNA were isolated from $100 \mathrm{mg}$ of roots, stems, leaves, petals and treated leaves and reverse transcribed to the first-strand cDNAs with an oligo (dT) primer according to the manual of PrimeScript ${ }^{\oplus}$ RT reagent Kit (TaKaRa, Japan). The qRT-PCR was performed on an ABI 7500 Real-Time PCR System with the primers of CsGPX2-F (5'-CCAGGAGCCAGGGAATAATGAG$3^{\prime}$ ) and CsGPX2-R (5'-GGAGCAGCATTCTCACCATT CA-3'). The 18SrRNA gene (18Sr RNA-F: 5' -CGGCTAC CACATCCAAGGAA-3', 18Sr RNA-R: 5'-GCTGGAAT TACCGCGGCT-3') was used as internal control gene in all qRT-PCR reactions above (Sun et al. 2010).

\section{Results and discussion}

\section{Cloning of full-length cDNA}

Two fragments, a 286 bp long 3 ' - flanking region with poly (A) and a 519 bp long 5' - untranslated region (UTR) were obtained by RACE method respectively. Both sequences showed high homology with glutathione peroxidase genes of angiosperms, and they were spliced to get full-length cDNA. The complete nucleotide sequence was denominated as tea plant glutathione peroxidase 2 (CsGPX2, Accession NO. JQ247186), because of an already known glutathione peroxidase gene named as CsGPX and according to the nomenclature used by Mullineaux et al. 1998 and Rodríguez Milla et al. 2003. The CsGPX2 gene was 917 bp long including a 5' - UTR of 265 bp and a 3'- UTR of $142 \mathrm{bp}$, and the ORF was $510 \mathrm{bp}$ with 169 deduced amino acids. The deduced amino acid sequence of CsGPX2 (CsGPX2) contained the motif of ILAFPCNQF and FTVKD, which were commonly conserved in glutathione peroxidase family. Using the online Computer $\mathrm{pI} / \mathrm{Mw}$ Tool http://cn.expasy.org/tools), the theoretical isoelectric point $(p I)$ and molecular weight $(\mathrm{MW})$ of CsGPX2 was calculated, and the two values were 7.62, $18.8 \mathrm{kDa}$ respectively.

\section{Sequence analysis and expression profiles in tea plant organs}

The deduced amino acid sequence of CsGPX2 comparison was performed by Blast P online (http://www.ncbi.nlm.nih. gov/). The protein sequence was most similar to glutathione peroxidases of angiosperms with a similarity of $85 \%$ to

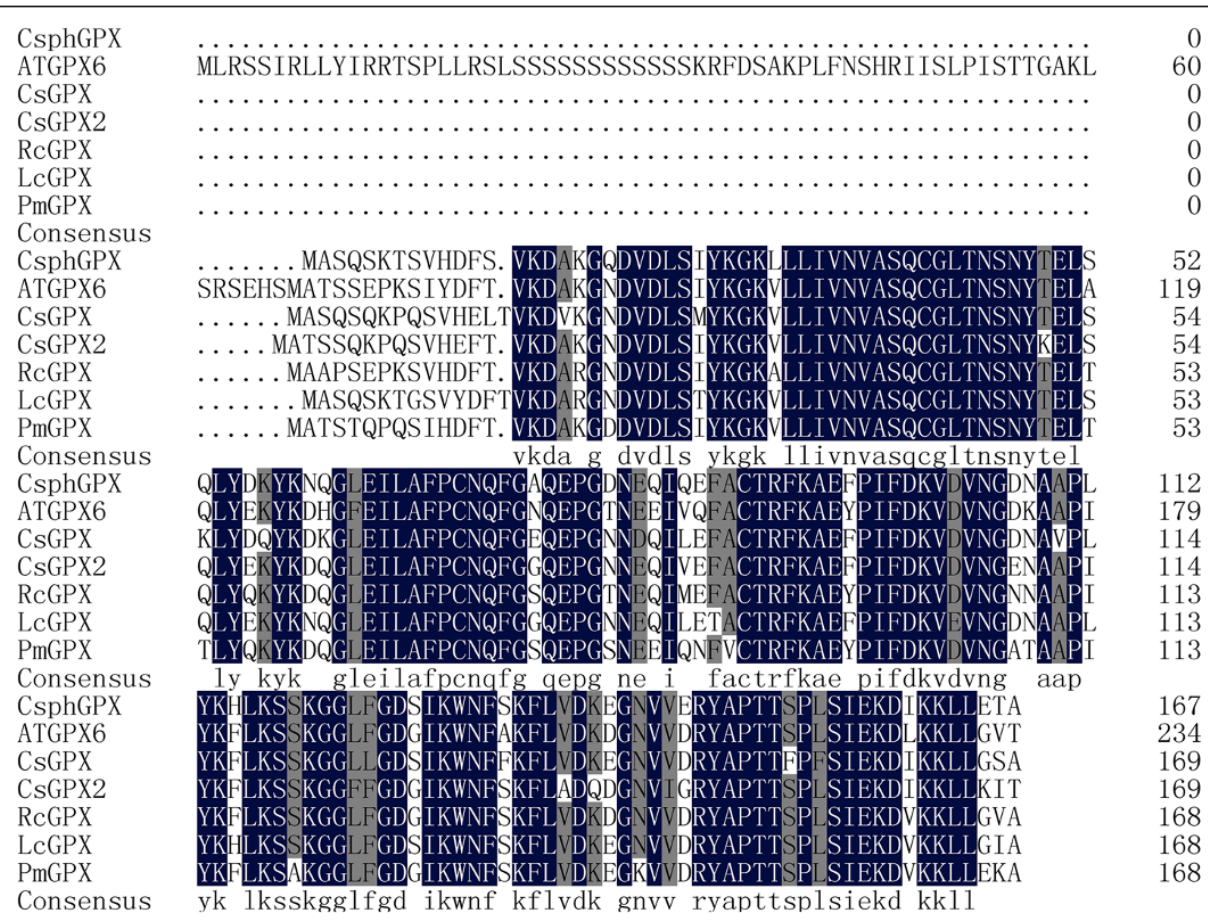

Figure 1 CsGPX2 (this work) sequence was aligned with several glutathione peroxidases. Camellia sinensis, CsGPX2; Ricinus communis, RcGPX, XP_002509790; Plantago major, PmGPX, CAJ43709; Litchi chinensis, LcGPX, ACl04528; Camellia sinensis, CsGPX, AEC10977, Citrus sinensis, CsphGPX, CAE46896; and Arabidopsis lyrata subsp. lyrata, ATGPX6, XP_002874703.1 were aligned. Conserved sequence elements are highlighted and identical residues were showed in the consensus line. Shading indicates levels of sequence conservation (100\% conservation: white on black; 75\% conservation: black on dark grey). 


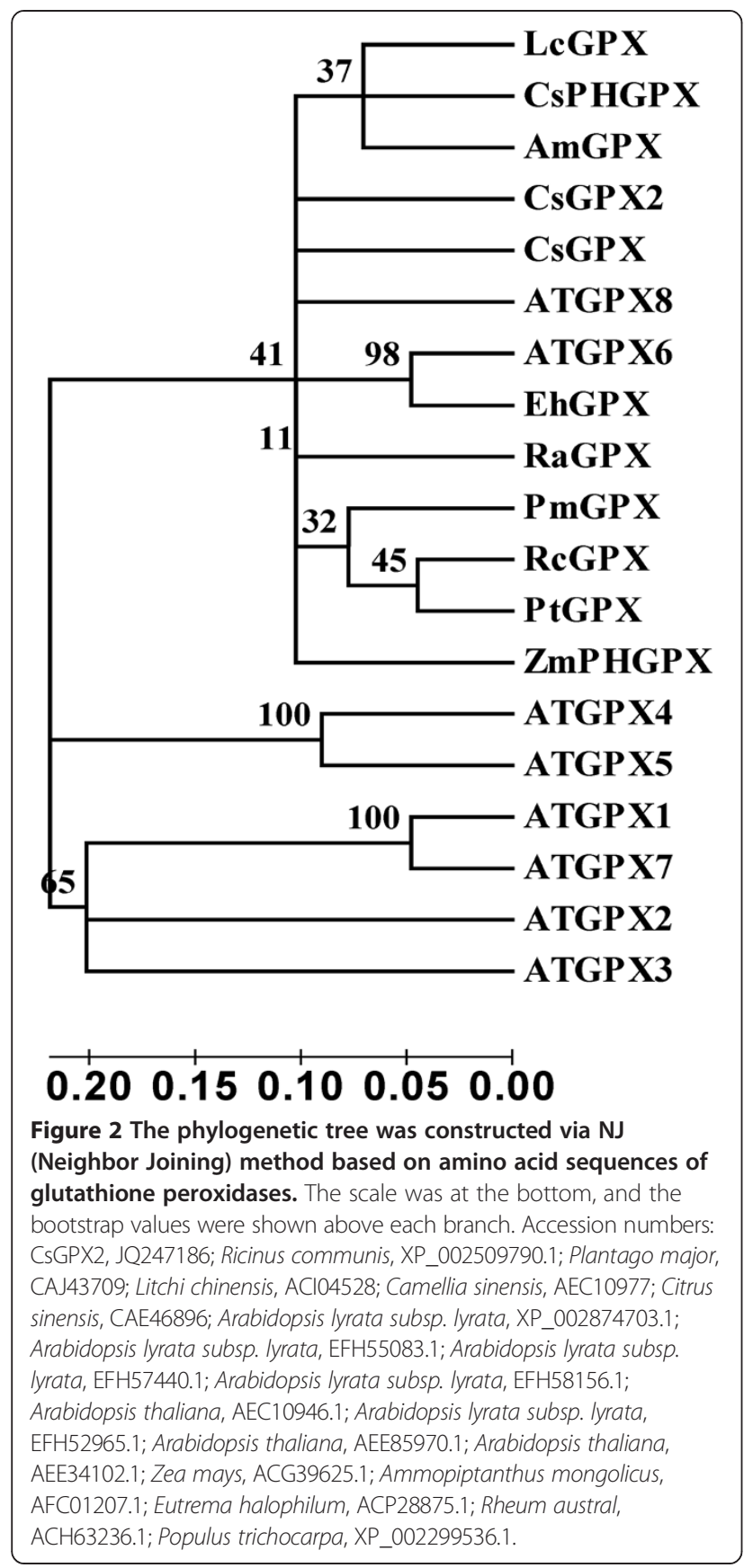

glutathione peroxidases from Ricinus communis, Arabidopsis lyrata subsp. lyrata, Populus trichocarpa (Accession NO. XP_002509790.1, XP_002874703.1, XP_002299536.1). While the similarity (84\%) with the known glutathione peroxidase gene of tea plant (Accession NO.AEC10977.1) was not as high as that of several genes above mentioned, which indicated that the two glutathione peroxidase genes CsGPX and CsGPX2 belonged to deferent clades (Figure 1). The sequence also showed high homologous with other characteristic conserved elements of glutathione peroxidases, including the most highly conserved metal

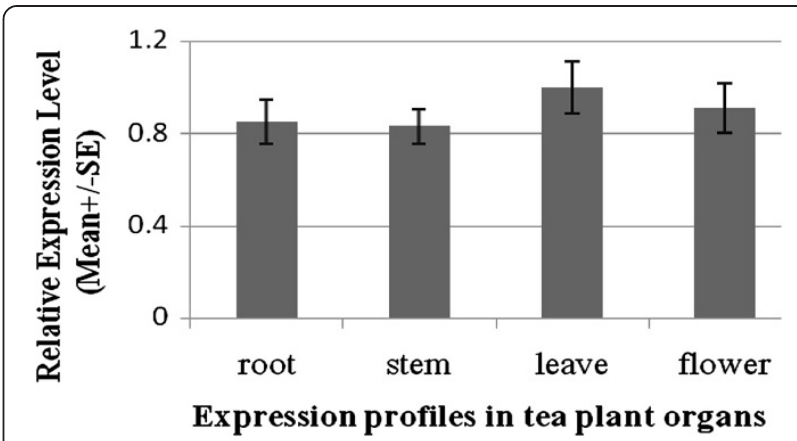

Figure 3 The relative expression levels of CsGPX2 in tea plant (Camellia sinensis) organs. Gene transcript in root, stem, leaf and flower were detected by qRT-PCR.

ion-binding motif of ILAFPCNQF. A phylogenetic tree was constructed based on the CsGPX2 sequence and other plant GPXs via CLUSTAL W2 online (http://www.ebi.ac. $\mathrm{uk} /$ ) and MEGA 4 software. The evolutionary relationship between CsGPX2 and selected sequences of higher plants was performed via NJ (Neighbor Joining) method, and the bootstrap values (1000 replicates) were shown above each branch (Figure 2). The phylogenetic tree clearly showed that GPXs from plants clustered in three groups, most GPXs including two gene of CsGPX2 and CsGPX from tea plant clustered one large group first, which suggested their divergence were recent with scales from 0.05 to 0.10 . Two Arabidopsis GPXs of ATGPX4 and ATGPX5 clustered a separate group, which indicated the evolution of these proteins within it was independent. Four genes of ATGPX1, ATGPX2, ATGPX3 and ATGPX7 clustered another group, and the divergence scales between the other two groups were both larger than 0.20 respectively.

The CsGPX2 expression levels in deferent organs of tea plant were investigated by real-time qRT-PCR. The relative expression level was $0.85,0.83,1.00$ and 0.91 in root, stem, leaf and flower respectively. This result showed

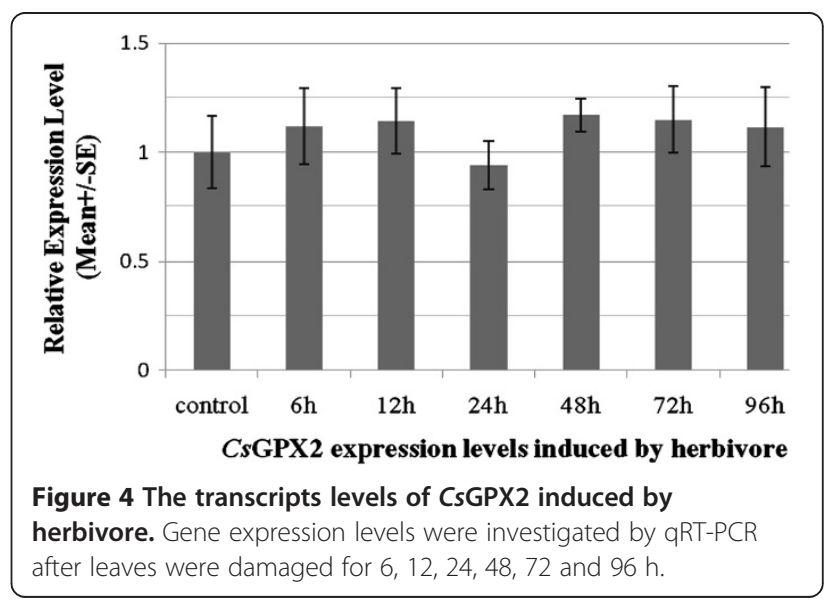


that CsGPX2 expressed at equivalent levels with no obvious difference in organs tested (Figure 3).

\section{CsGPX2 expression pattern under biotic and abiotic stresses}

To detect the gene expression profiles under biotic stress, seedlings were treated with starved tea geometrids, and the transcript levels were investigated by real-time qRT-PCR. Compared to the control, the gene relative expression level was $1.12,1.14,0.94,1.17,1.15$ and 1.11 respectively, after insect feeding for 6, 12, 24, 48, 72 and 96 hours. This result showed gene expression had no significant up-regulation or down-regulation, which indicated that CsGPX2 was not induced by herbivore damage (Figure 4). Real time qRTPCR was applied to analyze the gene expression levels under abiotic treatments of heavy metals, plant hormones, heat, drought and salt stress. The CsGPX2 transcript levels increased 1.8-, 2.9-, 1.9- and 3.1-fold under $\mathrm{Fe}^{2+}, \mathrm{Cu}^{2+}$, $\mathrm{NaCl}$ and mannitol treatments for 6 hours. Under heat treatment at $40^{\circ} \mathrm{C}$ for 3 hours, the gene expression increased 6.9-fold significantly. After hormone treatments for 12 hours, CsGPX2 was induced by GA and MeJA with 3.3- and 4.9-fold increase respectively, but the gene transcription was not sensitive to SA, ABA and NAA stresses (Figure 5).

The CsGPX2 expression was induced by abiotic stresses including salinity, heavy metal toxicity, drought and heat. While it was only induced dramatically by hormones of GA and MeJA, increased a little under ABA treatment,

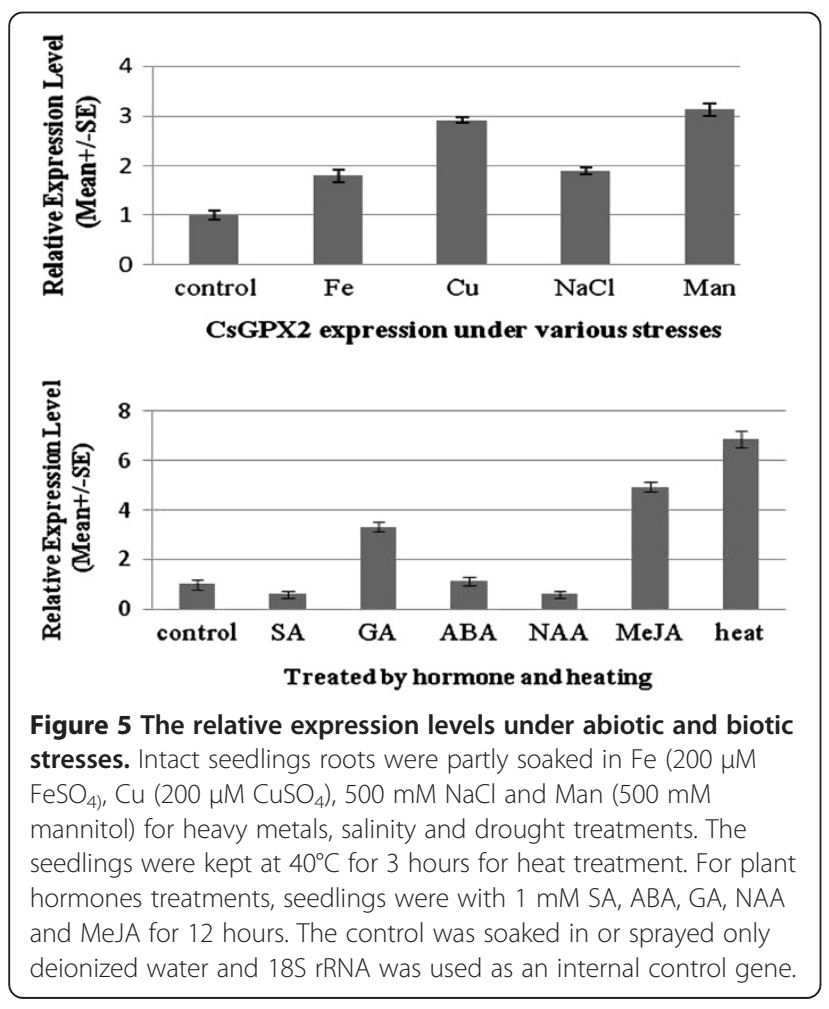

and even decreased a little under SA and NAA treatment. That suggested CsGPX2 expression level was more sensitive to heat, drought, heavy metal toxicity, but selectively to plant hormones with GA and MeJA. This result suggested that CsGPX2 could potentially function in protecting tea plant from oxidative damage under some abiotic stresses. Because CsGPX2 was not activated obviously under herbivore damage, the gene might have no function in biotic stress defense.

\section{Conclusion}

Glutathione peroxidase (GPX) is the general name for a family of isozymes that removes ROS using GSH as an electron donor (Margis et al. 2008; Wang et al. 2012). Generally, in plants GPXs localized at different cell organelles as mitochondria, chloroplast, endoplasmic reticulum/ cytosol or secreted (Ramos et al. 2009). The major two functions of GPXs in plants were to protect cell membranes from peroxidative damage and involved in redox transduction under stress (Gueta-Dahan et al., 1997; Miao et al. 2006; Gill and Tuteja 2010; Suzuki et al. 2011). When treated with stresses including salinity, heavy metal toxicity, drought, heat, cold and hormone, the expression of many GPX genes were enhanced dramatically (Holland et al. 1993; Avsian-Kretchmer et al. 2004; Ramos et al. 2009; Chang et al. 2009; Faltin et al. 2010).

In this paper, we successfully cloned a new gene designated as CsGPX2 encoding glutathione peroxidase from tea plant. Bioinformatics analysis showed that CsGPX2 contained the highly conserved metal ion-binding motif of ILAFPCNQF, and the amino acid sequence had high homology with GPXs of plants (Rodríguez Milla et al. 2003; Ramos et al. 2009). The CsGPX2 expression was induced by abiotic stresses including salinity, heavy metal toxicity, drought and heat, and similar results were observed in Arabidopsis and Citrus (Holland et al. 1993; Rodríguez Milla et al. 2003; Miao et al. 2006). The gene expression were only induced dramatically by hormones of GA and MeJA, increased a little under ABA treatment, and even decreased a little under SA and NAA treatment, which suggested that the gene expression was more sensitive to heat, drought, heavy metal toxicity, but selectively to plant hormones with GA and MeJA. Furthermore, because of CsGPX2 being not activated obviously under herbivore damage, the gene might have no function in biotic stress defense. Because those abiotic stresses lead to ROS production and CsGPX2 was significantly induced, which indicated that CsGPX2 may have potential function in protecting tea plant from peroxidative damage under abiotic stress.

In plants, most signal transduction pathways related to stress tolerance were divided into two types of dependent on ABA or ABA-independent (Shinozaki and YamaguchiShinozaki 1996). The result showed CsGPX2 was not 
induced by ABA, which suggested CsGPX2 was an ABAindependent gene in stress signal transduction of tea plant. The expression profiles showed CsGPX2 was ubiquitous in tea plant organs and regulated by different abiotic stresses, which indicated this gene contributed to the defense against oxidative damage caused by normal plant metabolism (Rodríguez Milla et al. 2003). These findings might help us to understand the high tolerance to heavy metals toxicity, mechanism of drought response and defense signal transduction way in tea plant.

\section{Competing interests}

The author declares that he has no competing interests.

\section{Acknowledgements}

This work was supported by the National Natural Science Foundation of China (Grant No. 31100503), the Fundamental Research Funds of National Nonprofit Research Institute for Tea Research Institute, CAAS, (Grant No. 2012ZL050), the Natural Science Foundation of Zhejiang province, China (Grant No. Y3080150), and the Key Innovation Team of Zhejiang Province (Grant No. 2011R09027-13)

Received: 5 November 2013 Accepted: 5 November 2013

Published: 18 January 2014

\section{References}

Agrawal GK, Rakwal R, Jwa NS, Agrawal VP (2002) Effects of signaling molecules, protein phosphatase inhibitors and blast pathogen (Magnaporthe grisea) on the mRNA level of a rice (Oryza sativa L.) phospholipid hydroperoxide glutathione peroxidase (OsPHGPX) gene in seedling leaves. Gene 283:227-236

Anjum NA, Ahmad I, Mohmood I, Pacheco M, Duarte AC, Pereira E, Umar S, Ahmad A, Khan NA, lqbal M, Prasad MNV (2012) Modulation of glutathione and its related enzymes in plants' responses to toxic metals and metalloids a review. Environ Exp Bot 75:307-324

Avsian-Kretchmer O, Gueta-Dahan Y, Lev-Yadun S, Gollop R, Ben-Hayyim G (2004) The salt-stress signal transduction pathway that activates the gpx 1 promoter is mediated by intracellular $\mathrm{H}_{2} \mathrm{O} 2$, different from the pathway induced by extracellular H2O2. Plant Physiol 135:1685-1696

Bae YA, Cai GB, Kim SH, Zo YG, Kong Y (2009) Modular evolution of glutathione peroxidase genes in association with different biochemical properties of their encoded proteins in invertebrate animals. BMC Evol Biol 9:72

Bailey-Serres J, Mittler R (2006) The roles of reactive oxygen species in plant cells. Plant Physiol 141:311

Bhattacharjee S (2012) The language of reactive oxygen species signaling in plants. J Bot, doi:10.1155/2012/985298

Chang CC, Ślesak I, Jordá L, Sotnikov A, Melzer M, Miszalski Z, Mullineaux PM, Parker JE, Karpińska B, Karpiński S (2009) Arabidopsis chloroplastic glutathione peroxidases play a role in cross talk between photo-oxidative stress and immune responses. Plant Physiol 150:670-683

Criqui MC, Jamet E, Parmentier Y, Marbach J, Durr A, Fleck J (1992) Isolation and characterization of a plant cDNA showing homology to animal glutathione peroxidases. Plant Mol Biol 18:623-627

Faltin Z, Holland D, Velcheva M, Tsapovetsky M, Roeckel-Drevet P, Handa AK, Abu-Abied M, Friedman-Einat M, Eshdat Y, Perl A (2010) Glutathione peroxidase regulation of reactive oxygen species level is crucial for in vitro plant differentiation. Plant Cell Physiol 7:1151-1162

Feng DJ, Tang Q, Ye SR, Cheng NW, Zhang SM, Tan HP (2009) Study on Compound Pollution Effects of $\mathrm{Pb}^{2+}, \mathrm{Cd}^{2+}$ and $\mathrm{Ni}^{2+}$ on Tea Plant. J Sichuan Agric Univ 27:184-188

Gaber A, Ogata T, Maruta T, Yoshimura K, Tamoi M, Shigeoka S (2012) The involvement of Arabidopsis glutathione peroxidase 8 in the suppression of oxidative damages in nucleus and cytosol. Plant Cell Physiol 53:1596-1606

Gill SS, Tuteja N (2010) Reactive oxygen species and antioxidant machinery in abiotic stress tolerance in crop plants. Plant Physiol Biochem 48:909-930

Gueta-Dahan Y, Yaniv Z, Zilinskas BA, Ben-Hayyim G (1997) Salt and oxidative stress: similar and specific responses and their relation to salt tolerance in citrus. Planta 203:460-469
Holland D, Ben-Hayyim G, Faltin Z, Camoin L, Strosberg AD, Eshdat Y (1993) Molecular characterization of salt-stress-associated protein in Citrus: protein and CDNA sequence homology to mammalian glutathione peroxidases. Plant Mol Biol 21:923-927

Hossain MA, Piyatida P, Teixeira da Silva JA, Fujita M (2012) Molecular Mechanism of Heavy Metal Toxicity and Tolerance in Plants: Central Role of Glutathione in Detoxification of Reactive Oxygen Species and Methylglyoxal and in Heavy Metal Chelation. J Bot 2012:1-37

Jung BG, Lee KO, Lee SS, Chi YH, Jang HH, Kang SS, Lee K, Lim D, Yoon SC, Yun DJ, Inoue Y, Cho MJ, Lee SY (2002) A chinese cabbage CDNA with high sequence identity to phospholipid hydroperoxide glutathione peroxidases encodes a novel isoform of thioredoxin-dependent peroxidase. J Biol Chem 277:12572-12578

Margis R, Dunand C, Teixeira FK, Margis-Pinheiro M (2008) Glutathione peroxidase family: an evolutionary overview. J FEBS 275:3959-3970

Miao YC, Bai L, Miao C, Chen J, Song CP (2005) Progress in plant glutathione peroxidase. Chin Bull Bot 22:350-356

Miao YC, Lv D, Wang PC, Wang XC, Chen J, Miao C, Song CP (2006) An Arabidopsis glutathione peroxidase functions as both a redox-transducer and a scavenger in abscisic acid and drought stress responses. Plant Cell 18:2749-2766

Mullineaux PM, Karpinski S, Jiménez A, Cleary SP, Robinson C, Creissen GP (1998) Identification of CDNAs encoding plastid-targeted glutathione peroxidase. Plant J 13:375-379

Navrot N, Collin V, Gualberto J, Gelhaye E, Hirasawa M, Rey P, Knaff DB, Issakidis E, Jacquot JP, Rouhier N (2006) Plant glutathione peroxidases are functional peroxiredoxins distributed in several subcellular compartments and regulated during biotic and abiotic stresses. Plant Physiol 142:1364-1379

Ramos J, Matamoros MA, Naya L, James EK, Rouhier N, Sato S, Tabata S, Becana M (2009) The glutathione peroxidase gene family of Lotus japonicus: characterization of genomic clones, expression analyses and immunolocalization in legumes. New Phytol 181:103-114

Rodríguez Milla MA, Maurer A, Rodríguez Huete A, Gustafson JP (2003) Glutathione peroxidase genes in Arabidopsis are ubiquitous and regulated by abiotic stresses through diverse signaling pathways. Plant J 36:602-615

Shinozaki K, Yamaguchi-Shinozaki K (1996) Molecular responses to drought and cold stress. Curr Opin Biotechnol 7:161-167

Sun M, Wang YS, Yang DQ, Wei CL, Gao LP, Xia T, Shan Y, Luo Y (2010) Reference genes for real-time fluorescence quantitative PCR in Camellia sinensis. Chin Bull Bot 45:579-587

Suzuki N, Koussevitzky S, Mittler R, Miller G (2011) ROS and redox signalling in the response of plants to abiotic stress. Plant Cell Environ 35:259-270

Ursini F, Maiorino M, Brigelius-Flohé R, Aumann KD, Roveri A, Schomburg D, Flohé L (1995) Diversity of glutathione peroxidases. Methods Enzymol 252:38-48

Wang F, Duan YX, Chen L, Wang YY, Zhu XF, Li W (2012) Glutathione Peroxidase CDNA Cloning and Expression in Soybean Roots under Heterodera glycines Infection. J Agric Sci 7:163-171

Yang XD, Dong CJ, Liu JY (2006) A plant mitochondrial phospholipid hydroperoxide glutathione peroxidase: its precise localization and higher enzymatic activity. Plant Mol Biol 62:951-962

\section{doi:10.1186/1999-3110-55-7}

Cite this article as: Fu: Cloning of a new glutathione peroxidase gene from tea plant (Camellia sinensis) and expression analysis under biotic and abiotic stresses. Botanical Studies 2014 55:7. 